\title{
Katja Novitskova
}

\section{Approximations}

E

volutionary morphogenesis - the emergence of living forms - has led to the continuous, immensely complex and varied life we find on Earth. Every part of the human physiology and limbic system has developed through gradients of biochemical reactions in response to the conditions our humanoid and nonhumanoid ancestors have endured over time. Our eyes, brain lobes and hands have all been shaped over eons to perceive and interact with the world or others inhabiting it.

It can be claimed that on a fundamental level art is a unique way of capturing human attention by creating a model, a novel approximation, of something that exists in the world or has a potential to exist. An ochre drawing of a deer on a cave wall is an abstraction of the animal's shape, and the lines that define it make up a pattern that activates our attention, recognition and emotion. Approximation and pattern making are both the form and content of art. Art history is full of animal representations.

I made the first works of my Approximation series in the early 2010 s as a response to a new visual culture arising with the dawn of smartphones and tablets. At the time, major social media companies had just introduced views, likes and engagement stats: these concepts quickly became eradefining and established massive economies based on the quantification of human attention. As a result it became easier to identify the flow and virality of images on the web: what kind of content is popular and how it spreads. It was obvious that certain categories of images were outperforming the rest: animals and baby animals, cats, babies doing funny things, beautiful people, jokes, etc. Increasingly with some species of animals, there might be more images of them in existence online than bodies in actuality in the wild.

Imagine a scene: a couple of penguin parents arching over their baby in a near-perfect symmetry in Antarctica; the light from the setting sun is reflecting gracefully on their feather colourations and their cute offspring is sitting between them. This iconic moment would have gone "unnoticed' by the world if not for the human photographer who captured and stored it on a technological eye. Another scene: a baby giraffe bonding with its mother; their faces leaning onto each other, forming a composition of almost divine beauty 


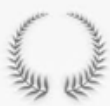
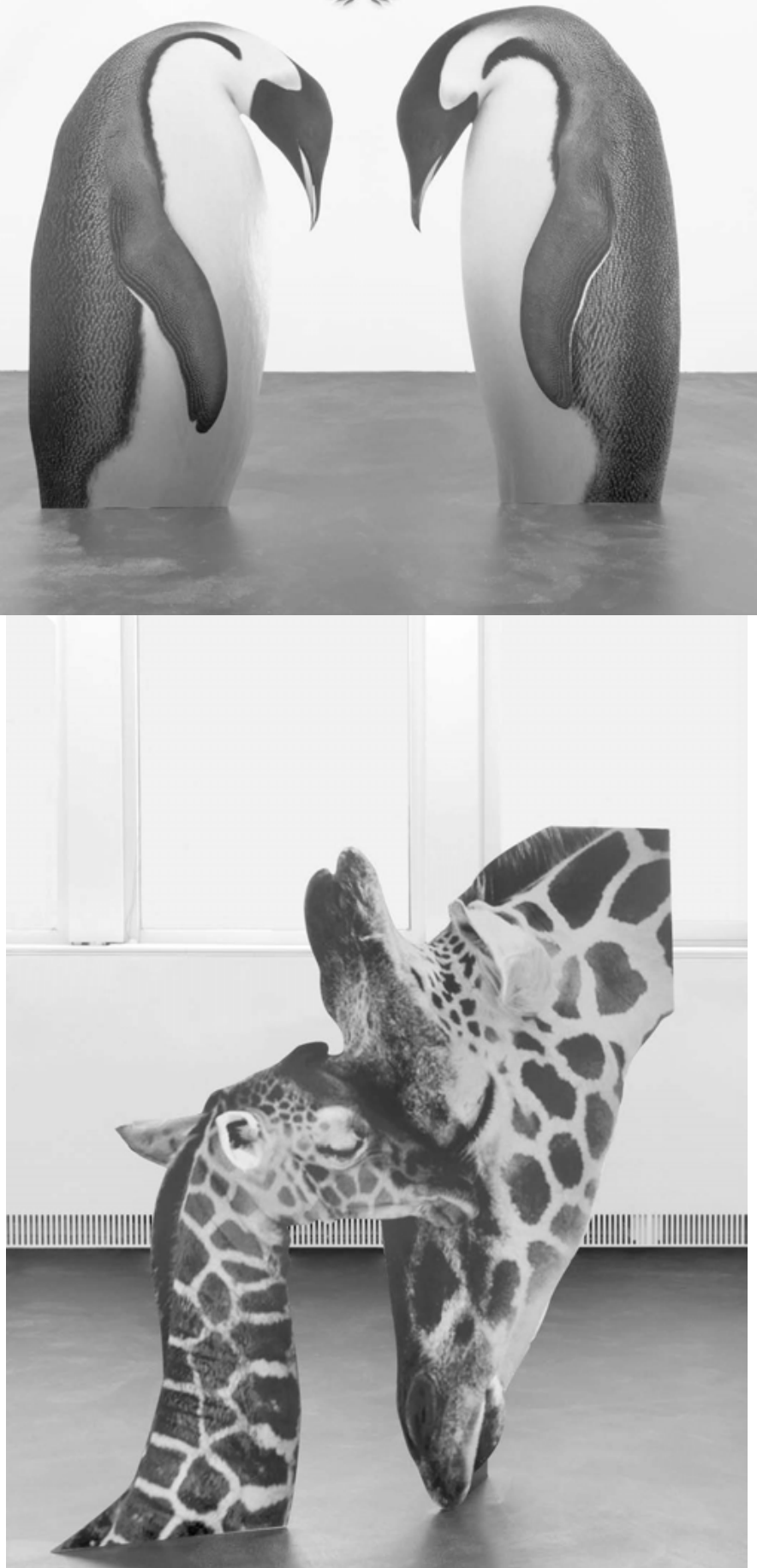
everyone in the world would recognise. The resulting photographs are approximations of these real life scenes. The animals turn into a signal, a pattern for our brains to process and enjoy and then trigger an emotional response: like and share.

Not all animals go viral. The ones that usually do fall under a few major aesthetic categories: cute or beautiful, alien or uncanny, funny looking or in a state of bonding. Cuteness is in itself a product of both evolutionary embryogenesis (babies of many species share specific visual characteristics like big eyes, short limbs, fluffy fur) and our psychological ability to identify and relate to cuteness across the interspecies boundaries. Products that base their designs on cuteness drive contemporary industries, and the Internet has amplified this greatly.

Beauty is more complex: it can be understood as an adaptive pattern complexity in nature, it can be understood as a social construct, it can be understood as something nonhuman or deeply human. It is clear though that beauty existed in the world way before the emergence of humans and our frontal lobes.

My experiment with the Approximation series consisted of figuring out if I can channel the attention and affect generated by the images of animals online towards the art works that use these images as material in real life. I take the signal of the animal forms out of the whole photograph, isolate and enlarge it, amplifying the intensity of the visual pattern. As photographic sculptural objects standing in gallery spaces they easily draw people's attention. This attention is then transformed into reactions that are perhaps similar to the ones triggered in people at the zoo or with a trophy: they take their phones and take a picture, or pose in front of the sculpture to be photographed. The affective reaction and documentation of the sculptures translated into likes on social media, generating a new form of attention-value and eventually helped me establish a career in the arts.

Another, more speculative, proposition I make with these works is that in order to conceptually model the reality of complex future technologies such as 'general AI', it might be productive to use animals and biological phenomena as approximations, both through their representations and their complexity. As a living feeling machine a tiny chameleon is still infinitely more intricate than a smartphone, a drone or hospitality robot. Looking anew at nonhuman sentience and biology in its various existing forms allows paths beyond anthropomorphising $\mathrm{AI}$ and beyond human hubris. Aesthetic, emotional and bio-technological complexities all go hand in hand. And so perhaps perceiving animal forms again through the transformative lens of art or poetry might help us create paradoxically realistic conceptual models of future complexities. In this framework the image of an animal can be recognised as representing something else entirely. 

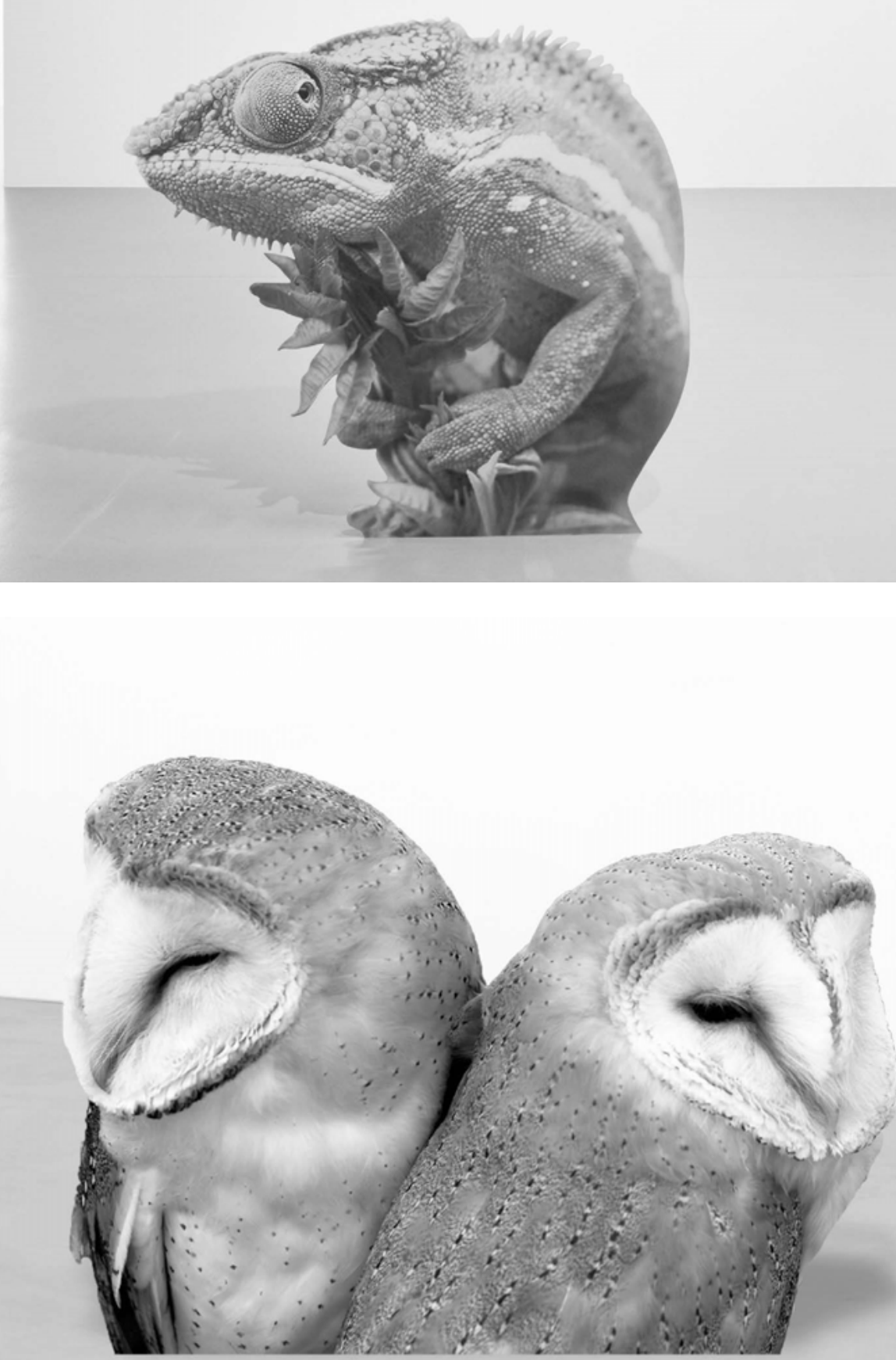


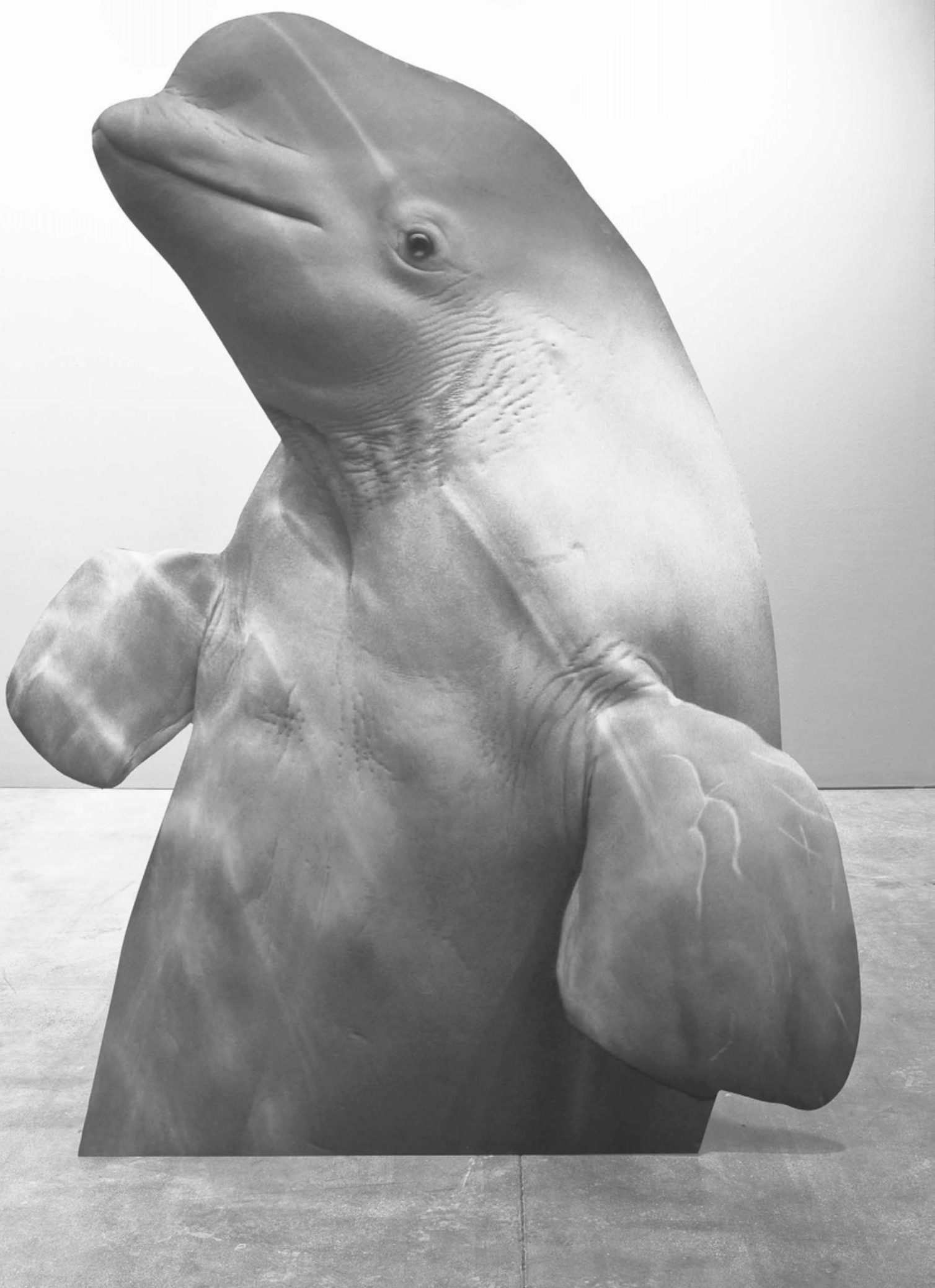



(25)

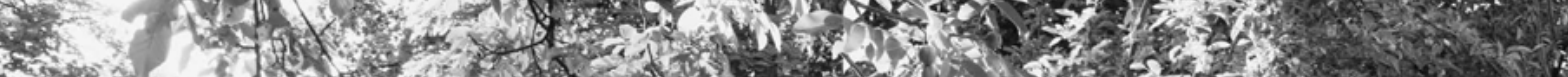

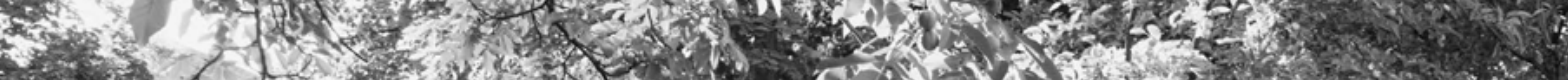

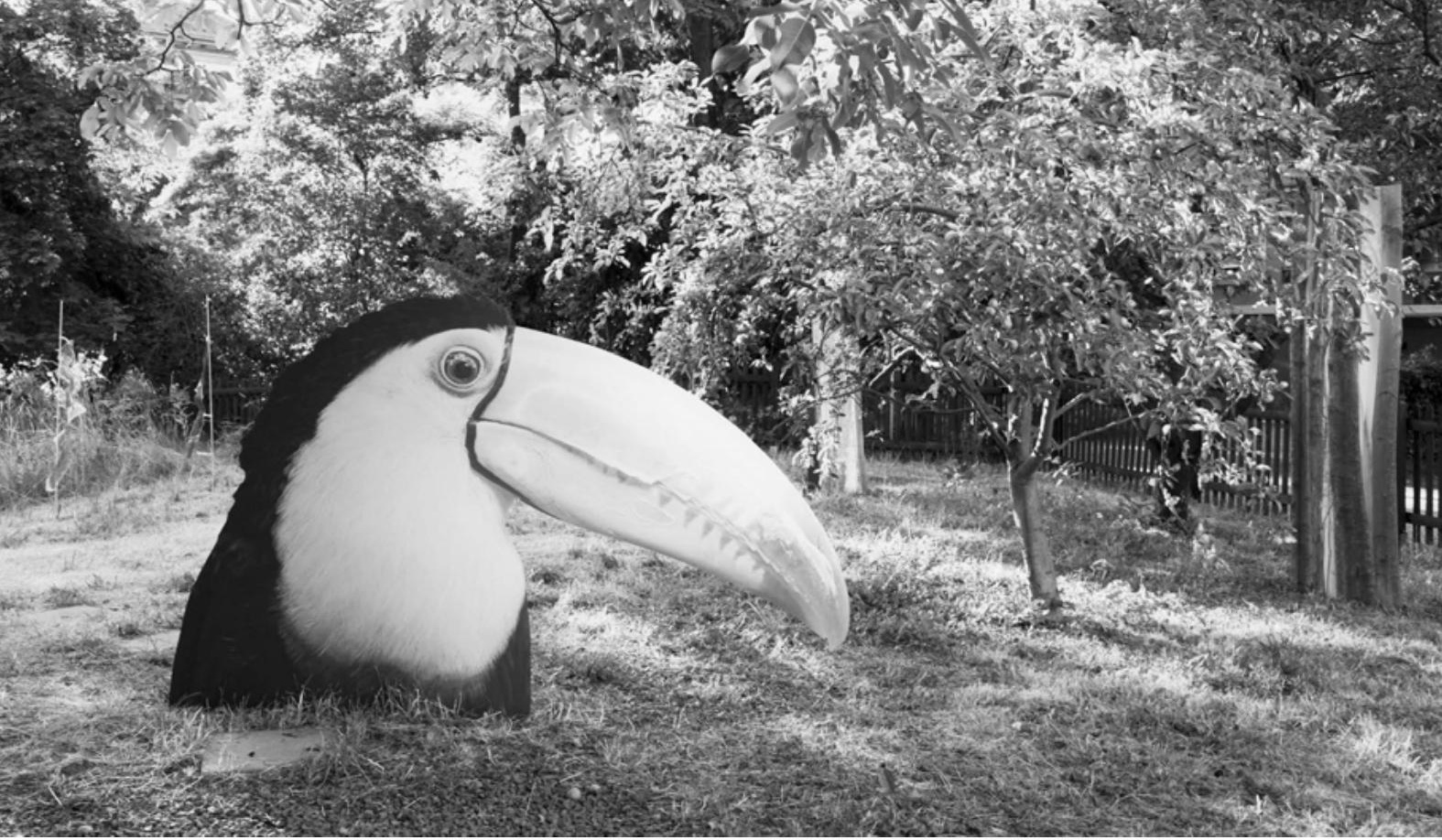

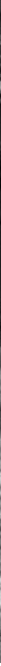

